\title{
Antibody pincer movement challenges sunitinib as standard therapy
}

Results of a recently published phase III trial show that overall survival and objective response rate (ORR) were significantly higher in patients with advanced intermediate-risk or poor-risk renal cell carcinoma (RCC) treated with nivolumab plus ipilimumab than in those receiving sunitinib.

The tyrosine kinase inhibitor (TKI) sunitinib is a first-line treatment for patients with advanced RCC, providing an ORR of $\sim 25 \%$, but haematological toxicity is common. The antibodies nivolumab and ipilimumab are immune checkpoint inhibitors (ICIs) that target programmed cell death protein 1 (PD1) and cytotoxic T lymphocyte antigen 4 (CTLA4) and are approved as single agents for the treatment of advanced RCC and melanoma, respectively. "Based on the mechanism of action of nivolumab and ipilimumab and data from previous trials, it made sense to evaluate the combination treatment in a phase III trial in patients with metastatic RCC," Padmanee Sharma (MD Anderson Cancer Center, Texas, USA), one of the corresponding authors, tells Nature Reviews Urology.

A total of 1,096 patients, of whom $61 \%$ had intermediate-risk and $16 \%$ had poor-risk disease, were randomly assigned to receive nivolumab plus ipilimumab or sunitinib. In this population with elevated risk, 18-month and median overall survival were $75 \%$ and not reached, respectively, for nivolumab plus ipilimumab versus $60 \%$ and 26.0 months, respectively, for sunitinib after a median follow-up period of 25.2 months. The hazard ratio for death was $0.63(99.8 \% \mathrm{CI}$ $0.44-0.89 ; P<0.001)$. The ORR in these patients was $42 \%$ for the ICIs and $27 \%$ for the TKI $(P<0.001)$;

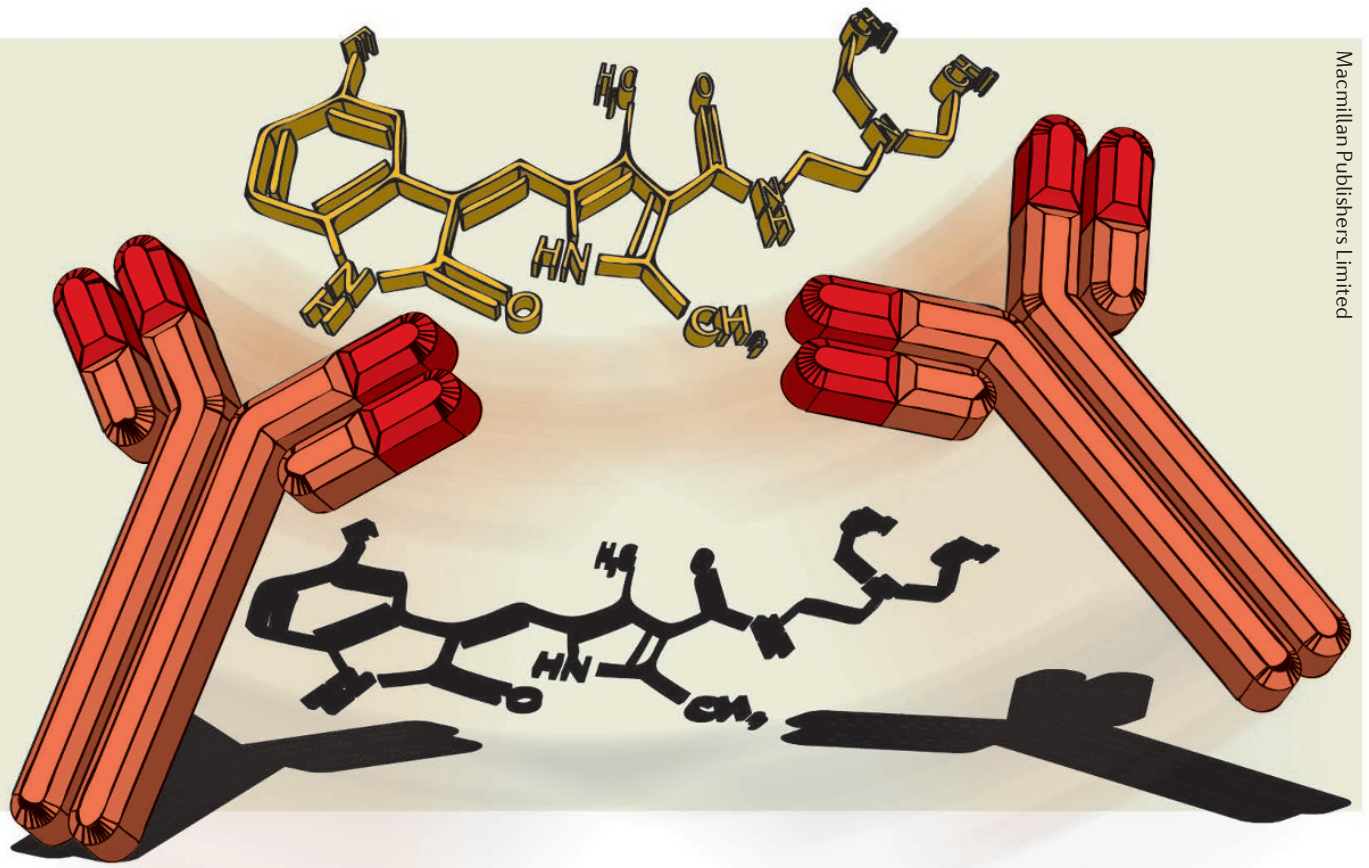

complete responses occurred in 40 patients and 5 patients, respectively. Subgroup analyses showed favourable overall survival and ORR for the ICIs across patients independent of age, sex, previous nephrectomy, PD1 ligand expression status, or metastases location.

Analysis of patients in all risk groups showed an 18-month and median overall survival of $78 \%$ and not reached, respectively, for the ICIs versus $68 \%$ and 32.9 months, respectively, for the TKI (HR 0.68; $99.8 \% \mathrm{CI}$ $0.49-0.95 ; P<0.001)$. In the ICI and TKI groups, $46 \%$ and $63 \%$ of patients had grade 3 or 4 treatment-related adverse events and $22 \%$ and $12 \%$ of patients discontinued treatment owing to adverse events, respectively.

"The combination therapy improved patient survival, which provides rationale for approval as a first-line treatment for metastatic RCC, but some patients' disease still progressed," comments Sharma. "Our efforts focus on understanding why only some patients respond. Other combinations might be worth considering, such as ICIs plus other TKIs or other novel agents, possibly targeting macrophages or dendritic cells to engage innate immune responses, which might generate favourable clinical responses for even more patients. We're currently evaluating triplet combination of nivolumab plus bevacizumab plus metastasectomy as one arm of therapy in a pilot study treating patients with metastatic RCC."

Clemens Thoma

ORIGINAL ARTICLE Motzer, R. J. et al. Nivolumab plus ipilimumab versus sunitinib in advanced renal-cell carcinoma. N. Engl.J. Med. https://doi.org/10.1056/NEJMoa1712126 (2018) 